\title{
The Velocity Distribution of Wind Currents in the Eastern Part of the Equatorial Pacific.
}

\author{
By \\ K. KAJIURA \\ (Geophysical Institute, Tokyo University)
}

\begin{abstract}
Wind driven currents in a baroclinic ocean are discussed following the ideas of SVERDRUP and REID, and the current velocities in the upper layers of the eastern equatorial Pacific are calculated on the assumption that the inertial forces and the lateral friction are negligible. According to the present theory, currents may be devided into two parts, of which one is the pure drift currents and the other is the relative currents, and it is found that the results are in good agreement with the present knowledge of the currents near the equator. In these regions, the relative currents are much stronger than the pure drift currents and the north-south component of the velocity is very small compared with the east-west component.
\end{abstract}

\section{Introduction.}

Recent theories on the wind driven ocean circulation have shown the circulation in the upper layers of the oceans being the result chiefly of the stresses exerted by winds. The stream lines of oceanic mass transport derived from solutions of a vertically integrated vorticity equation account for many of the gross features of the general circulation (MuNK, 1950).

Mass transport methods, however, add only little to the knowledge of the vertical structure of currents. In this respect, Hidaka (1950) treated this problem by using the velocity components themselves instead of the mass transport, but the results were not satisfactory. Different approach to this problem is attempted here which may be successful in a region where lateral stresses of the currents can be neglected.

According to the ideas of SverdRup (1947) and REID (1948), the pressure field in a baroclinic ocean can be computed by the knowledges of the wind stresses and the vertical structure of water-mass. Therefore the problem to be solved is reduced to find currents under the influence of wind stresses in the known pressure field. This problem is similar to that discussed by EKMAN (1906).

\section{Theory.}

In a region where lateral stresses and field accelerations can be neglected, the equations of stationary motion of water can be written; 


$$
\begin{array}{r}
2 \rho \omega \cdot v \sin \varphi+\mu \frac{\partial^{2} u}{\partial z^{2}}-\frac{\partial p}{\partial x}=0, \\
-2 \rho \omega \cdot u \sin \varphi+\mu \frac{\partial^{2} v}{\partial z^{2}}-\frac{\partial p}{\partial y}=0 .
\end{array}
$$

Here $u$ and $v$ are the $x$ and $y$ components of velocity where $x$ - and $y$-axes are directed toward the east and north, respectively, with the origin on the equator, and the $z$ - axis is taken to be positive downwards. And $\varphi$ is the latitude, taken positive to the north of the equator, $\omega$, the angular velocity of the Earth's rotation, $\rho$, the density, $\not$, the pressure and $\mu$, the eddy viscosity.

Writing

$$
W=u+i v, \quad \alpha=\sqrt{\frac{\rho \omega \sin \phi}{\mu}} \text { and } F=\frac{1}{\mu}\left(\frac{\partial p}{\partial x}+i \frac{\partial p}{\partial y}\right)
$$

where $i^{2}=-1$, the equations (1) are reduced to,

$$
\frac{\partial^{2} W}{\partial z^{2}}-2 \alpha^{2} i W=F \text {. }
$$

In this equation $\alpha$ includes $\rho$ and $\mu$ both of which must be functions of $z$. The variation of the density with depth, however, is too small to modify the results and the knowledges of the eddy viscosity are not enough at present to draw reliable conclusions as to the variation of $\alpha$ with depth. Therefore, on the assumption of $\alpha$ to be constant in the vertical direction, the general solution of the equation (2) is easily obtained as follows:

$$
\begin{aligned}
W=A e^{\alpha(1+i) z}+B e^{-\alpha(1+i) z} & +\frac{e^{\alpha(1+i) z}}{2 \alpha(1+i)} \int F e^{-\alpha(1+i) z} d z \\
& -\frac{e^{-\alpha(1+i) z}}{2 \alpha(1+i)} \int F e^{\alpha(1+i) z} d z,
\end{aligned}
$$

where $A$ and $B$ are arbitrary constants.

Introducing the boundary conditions such that $-\mu \frac{\partial W}{\partial z}=T$ at the surface and $W=0$ at a sufficiently great depth, in which $T=\tau_{x}+i \tau_{y}$ and $\tau_{x}$ and $\tau_{y}$ are $x$ and $y$ components of the wind stresses, $W$ can be determined definitely when $F$ is known.

In a baroclinic ocean it is reasonable to assume the horizontal velocity and the horizontal pressure gradient vanish at a moderate depth below the sea surface. If $d$ is the selected depth of no motion, the vertically integrated equations of motion from the surface to the depth, $d$, on the assumption that $\frac{d u}{d z}=\frac{d v}{d z}=0$ at the depth, $d$, are

$$
\begin{aligned}
2 w \sin \varphi \cdot M_{y}+\tau_{x}-\frac{\partial P}{\partial x} & =0, \\
-2 \omega \sin \varphi \cdot M_{x}+\tau_{y}-\frac{\partial P}{\partial y} & =0 .
\end{aligned}
$$


where $\quad M_{x}=\int_{-\zeta}^{a} \rho u d z, \quad M_{y}=\int_{-\zeta}^{a} \rho v d z$ and $P=\int_{-\zeta}^{a} p d z$.

The equation of continuity is,

$$
\frac{\partial M_{x}}{\partial x}+\frac{\partial M_{y}}{\partial y}=0 \text {. }
$$

By cross-differentiation of the two equations of (4) with the aid of (5), it follows that

$$
M_{y}=-\frac{R}{2 \omega \cos \phi} \cdot \frac{\partial \tau_{x}}{\partial y},
$$

where $\tau_{x}$ and $\tau_{y}$ are assumed to be independent of $x$, and $\varphi$ is taken to be $y / R$, in which $R$ is the radius of the earth.

Substitution of (6) into (5) leads to

$$
M_{x}=\frac{x-x_{0}}{2 \omega \cos \varphi}\left(\tan \varphi \frac{\partial \tau x}{\partial y}+R \frac{\partial^{2} \tau x}{\partial y^{2}}\right)
$$

Here the continental boundary is assumed at $x=x_{0}$ where no mass transport is present across the boundary.

By insertion of both (6) and (7) into (4) the following equations are obtained,

$$
\begin{aligned}
& \frac{\partial P}{\partial x}=\tau_{x}-R \tan \varphi \frac{\partial \tau_{x}}{\partial y}, \\
& \frac{\partial P}{\partial y}=-\left(x-x_{0}\right) \tan \varphi\left(\tan \varphi \frac{\partial \tau_{x}}{\partial y}+R \frac{\partial^{2} \tau_{x}}{\partial y^{2}}\right)+\tau_{y} .
\end{aligned}
$$

Assuming the Reid's model of the vertical structure of mass, such that $\rho=\rho_{0}$ at $-\zeta<z<h, \rho=\rho_{d}-\Delta \rho e^{1-z / h}$ at $h \leq z<d$ and $\rho=\rho_{d}$ at $d \leq z$, where $\Delta \rho=\rho_{d}-\rho_{0}$ and $-\zeta$ is the elevation of the sea surface, the pressure at any depth may be deternined by a integration of the hydrostatic equation.

From the condition that the pressure gradient vanishes below a moderate depth, it follows that,

$$
\frac{\partial \zeta}{\partial x} \fallingdotseq \frac{2 \Delta \rho}{\rho_{0}} \frac{\partial h}{\partial x} \text { and } \frac{\partial \zeta}{\partial y} \fallingdotseq \frac{2 \Delta \rho}{\rho_{0}} \frac{\partial h}{\partial y},
$$

when the depth of no motion is assumed to be sufficiently great compared with the depth of the upper homogeneous layer.

By use of the above equations (9), the following relations are obtained,

$$
\frac{\partial P}{\partial x}=5 g \Delta \rho h \frac{\partial h}{\partial x} \text { and } \frac{\partial P}{\partial y}=5 g \Delta \rho h \frac{\partial h}{\partial y},
$$

and from the equations (8) and (10), it follows that

$$
h^{2}=\frac{2}{5 g \Delta \rho}\left\{\left(x-x_{0}\right)\left(\tau_{x}-\frac{\partial \tau_{x}}{\partial y} R \tan \varphi\right)+\int^{y} \tau_{y} d y\right\}+C
$$

where $C$ is a constant of integration. 
The pressure distributions in the ocean are

$$
\begin{array}{ll}
\frac{\partial p}{\partial x}=g \rho_{0} \frac{\partial \zeta}{\partial x} & \text { at }-\zeta \leq z<h \\
\frac{\partial p}{\partial x}=g\left[\rho_{0} \frac{\partial \zeta}{\partial x}+\Delta \rho \frac{\partial h}{\partial x}\left\{(1+z) e^{-z / h}-2\right\}\right] \text { at } h \leq z<d
\end{array}
$$

and

$$
\frac{\partial p}{\partial x}=0 \quad \text { at } d \leq z .
$$

Similar expressions hold for $\partial p / \partial y$.

If the equations $(8,(9),(10),(11)$ and (12) are combined together, the pressure gradient at any depth can be computed from the wind stresses alone.

Formulae for the current velocities in the upper layers.

The arbitrary constants $A$ and $B$ in the equation (3) can be easily determined from the boundary conditions as the horizontal pressure gradient does not vary with depth in the upper homogeneous layer and it vanishes at the depth, $d$. For the sake of simplicity, the lower boundary is replaced from $z=d$ to $z=\propto$. This simplification may not greatly modify the result.

The current velocities in the upper layers are given by,

and

$$
u=\frac{e^{-\alpha z}}{\sqrt{2} \mu \alpha}\left\{\tau x \sin \left(\frac{\pi}{4}-a z\right)+\tau_{y} \cos \left(\frac{\pi}{4}-a z\right)\right\}-\frac{1}{2 \mu \alpha^{2}} \frac{\partial p}{\partial y},
$$

$$
v=\frac{e^{-\alpha z}}{\sqrt{2} \mu \alpha}\left\{\tau_{y} \sin \left(\frac{\pi}{4}-a z\right)-\tau_{x} \cos \left(\frac{\pi}{4}-a z\right)\right\}+\frac{1}{2 \mu \alpha^{2}} \frac{\partial p}{\partial x},
$$

where $\frac{\partial p}{\partial x}=\frac{2}{5 h} \frac{\partial P}{\partial x}$ and $\frac{\partial p}{\partial y}=\frac{2}{5 h} \frac{\partial P}{\partial y}$ at $-\zeta \leq z<h$.

In the equations (13) the first term of the right hand side represents the pure drift current similar to that discussed by Ekman and the second term represents the relative current which depends on the distribution of density.

The calculation of current velocities.

The density distribution being related to the stress gradient, it is desirable to determine the accurate distribution of wind stresses, but it is impossible at present because of the lack of available observations. Therefore, we assume the sinusoidal stress distribution in the equatorial region of the North Pacific similar to the one assumed by Reid (1948), such that

$$
\tau_{x}=-\tau_{0}+b \sin k\left(\boldsymbol{\phi}-\varphi_{0}\right),
$$

and

$$
\tau_{y}=-b^{\prime} \sin k^{\prime}\left(\varphi-\varphi_{0}^{\prime}\right) \text {, }
$$


where $\tau_{0}=-0.3 \mathrm{dyne} / \mathrm{cm}^{2}, \quad b=0.2 \mathrm{dyne} / \mathrm{cm}^{2}, k=13.85, \phi_{0}=1^{\circ}, \quad b^{\prime}=0.4 \mathrm{dyne} / \mathrm{cm}^{2}$, $k^{\prime}=6.923$, and $\varphi_{0}{ }^{\prime}=7.5^{\circ}$. This formula for $\tau_{y}$ is applied only to the north of $4^{\circ} \mathrm{N}$ and it is assumed that $\tau_{y}$ decreases linearly from $4^{\circ} \mathrm{N}$ to the equator and it vanishes in the south latitude.

Numerical values used in calculation are as follows;

$$
\begin{aligned}
& \rho_{0}=1.0230, \quad \rho_{d}=1.0274, \quad d=700 \mathrm{~m}, \quad g=980 \mathrm{C} . \mathrm{G} . \mathrm{S} . \quad \omega=0.729 \times 10^{-4} / \mathrm{sec}, \\
& R=6400 \mathrm{Km}, \quad\left(x-x_{0}\right)=6500 \mathrm{Km}, \quad \mu=10^{2} \mathrm{~g} / \mathrm{cm} / \mathrm{sec} \text { and } C=2.8 \times 10^{8} .
\end{aligned}
$$

Fig. 1 is the vector representation of the wind stress and Fig. 2 shows the variation of currents with latitude. The drift current, depending on the absolute value of the wind stresses, varies its intensities according to the value of $\tau_{y}$, while the relative current which is mainly determined by the gradient of $\tau x$ in the north-south direction is only little affected by the presence of $\tau_{y}$ except near the coastal region.

Fig. 3 shows the velocity distribution in the upper layers. The drift current decreases exponentially with depth and the depth of frictional influence depends on the latitude and the value of eddy viscosity, so that the currents at the depth deeper than about $100 \mathrm{~m}$ are almost negligible except in the very vicinity to

Fig. 1.

The vector representation of the wind stresses in the Equatorial Pacific.

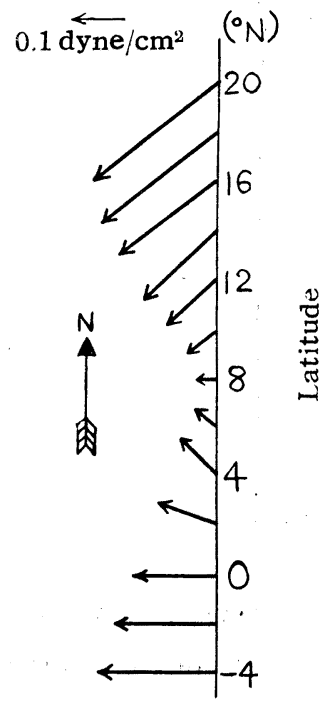

Fig. 2. The velocity distribution of the surface currents.

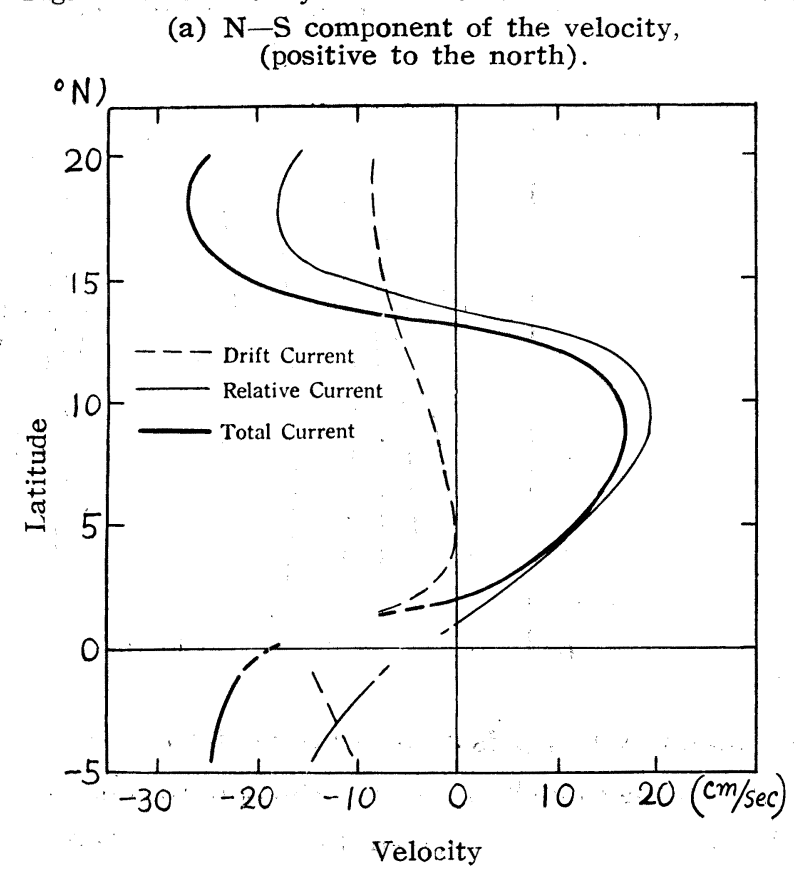


Fig. 2. The velocity distribution of the surface currents.

(b) E-W component of the velocity, (positive to the east).

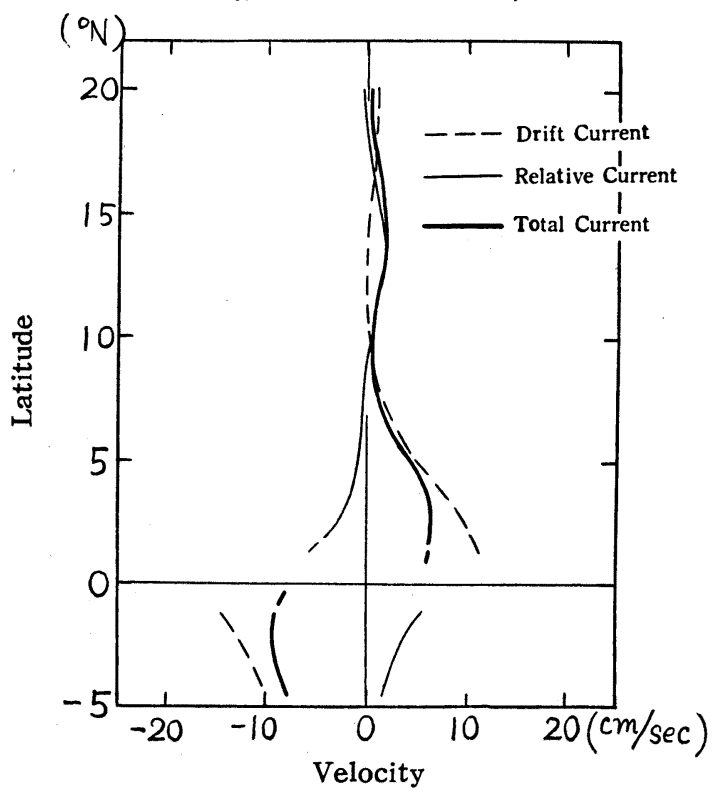

Fig. 3. The velocity distribution in the $\mathrm{N}-\mathrm{S}$ vertical section of the upper homogeneous layer (velocity, positive to the east, $\mathrm{cm} / \mathrm{sec}$ ).

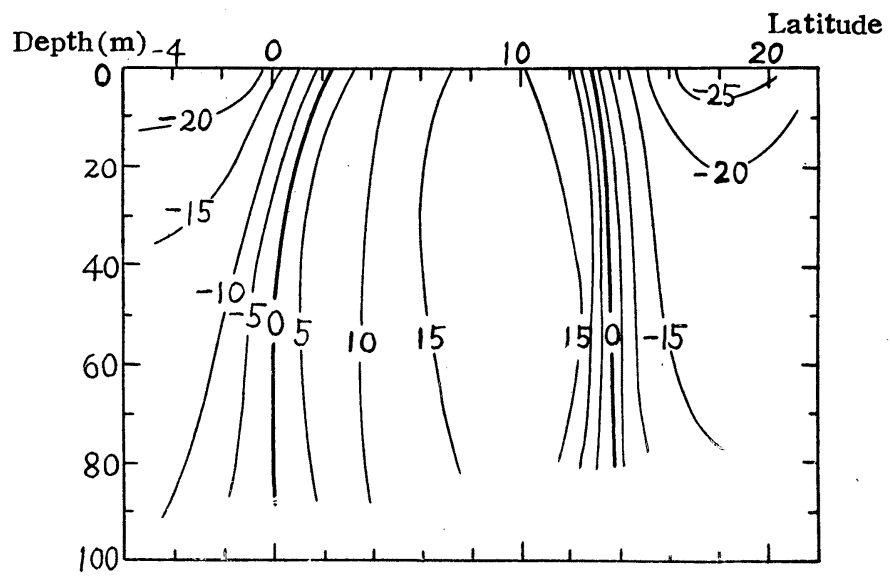

the equator, when $\mu$ is assumed to be $10^{2}$ C.G.S. In the surface layer, the drift current seems to make narrower the counter current and the maximum velocities of the counter current appear in the sub-surface layer. 
The maximum calculated velocity of the counter current is about $20 \mathrm{~cm} / \mathrm{sec}$ which is only half of the expected value of the velocity of the counter current. This discrepancy may be ascribed to the under-estimate of the wind stress in this region, or the neglect of the thermodynamic effect. On the whole, relative currents prevail compared with the drift currents. This conclusion is, however, not always reasonable as the drift current is developed by the temporary wind but the relative current is maintained by the stationary wind stress and is influenced only little by the temporary wind.

Just under the equator, the above theory fails and other factors such as lateral stresses, field accerelations and the thermodynamic processes must be taken into consideration.

Divergence and convergence of currents on the surface can be computed from the following equation;

$$
\frac{\partial u}{\partial x}+\frac{\partial v}{\partial y}=\frac{\partial p}{\partial x} \frac{\partial}{\partial y}\left(\frac{1}{2 \alpha^{2} \mu}\right)+\frac{\partial}{\partial y}\left(\frac{\tau_{y}-\tau_{x}}{2 a_{\mu}}\right)
$$

The calculation shows that no remarkable regions of divergence or convergence exist, except in the very vicinity of the equator.

\section{Concluding remarks.}

In the region discussed in this paper, the neglect of lateral stresses and inertia terms may not introduce serious result, because the horizontal stresses are rather small compared with the vertical stresses, even when the lateral eddy viscosity is taken to be $10^{8} \mathrm{c} . \mathrm{g} . \mathrm{s}$. except along the boundary line between the equatorial current and the counter current, where both stresses may become equal in magnitude.

When the drift current and the relative current are co-existing, the stationary state cannot be reached in a baroclinic ocean without some mechanism which re-establishes the distribution of density of water disturbed by drift currents. Lateral mixing processes and thermodynamic processes may be the factors which take part in this mechanism. In the present paper, however, the assumption of homogeneous water in the upper layer diminishes these difficulties and simplifies the treatment of phenomena.

Further investigations about the model of a water-mass are desirable but it seems very difficult to treat it exactly. The formation of water-mass is chiefly the result of thermodynamic processes such as evaporation and precipitation. If the thermodynamic processes act uniformly over the large area without any dynamic disturbances, a water-mass is formed in which the density of water 
may become a function of the depth only. When wind begins to blow under this circumstance and when the unequality of the wind velocity exist, the surface water is accumulated somewhere, resulting in the cause of the horizontal pressure gradient. This pressure gradient, reaching to the very bottom of the ocean, may produce the movement of the water in deep layers which changes the distribution of density. Thus the horizontal pressure gradient caused by the surface elevation will be cancelled soon by the re-distribution of density, and when the stationary state is reached, the water in the deeper layers will be at rest. In these ways the inner pressure gradient which becomes negligible at a moderate depth, may be generated.

The present paper is only a first step to study this interesting problem of the wind current in a baroclinic ocean, and many objections may be raised against the looseness of the treatments. Nevertheless we believe that the density distribution in the ocean may play an important role for the distribution of velocity of the wind current.

\section{Acknowledgement.}

The author wishes to express his sincere thanks to Prof. K. Hidaka for his support and encouragement during this work.

\section{References.}

Ekman, V. W. (1906) : Beiträge zur Theorie der Meeresströmungen. Ann. d. Hydro. u. Marit. Meteorol., 34, pp. 566-583.

Hidaka, K. (1950) : Drift Currents in an Enclosed Ocean, Part 1. Goophysical Notes (Geophysical Institute, Tokyo Univ.), 3, No. 23.

Munk, W. H. (1950) : On the Wind-Drived Ocəan Circulation. J. Meteorol., 7, pp. 79-93.

Reid, R.O. (1948a) : The Equatorial Curr nnts of the Eastern Pacific as Mainta ned by the Stress of the Wind. J. Marine Res., 7, pp. 74-99.

Reid, R.O. (1948b) : A Model of the Vertical Structure of Mass in Equatorial WindDriven Currents of a Baroclinic Ocean. J. Marine Res., 7; pp. 304-312.

Sverdrup, H. U. (1947) : Wind-Driven Currents in a Baroclinic Ocean; with Application to the Equatorial Currents of the Eastern Pacific. Proc. Nat. Acad. Sci., 33, pp. 318-326. 